\title{
Decreased expression of SLC39A I4 is associated with tumor aggressiveness and biochemical recurrence of human prostate cancer
}

This article was published in the following Dove Press journal:

OncoTargets and Therapy

II July 2016

Number of times this article has been viewed

\author{
Xiao-Ming $X u^{1,2}$ \\ Cheng-Gong Wang' \\ Yu-Di Zhu \\ Wei-Hua Chen ${ }^{3}$ \\ Si-Liang Shao ${ }^{2}$ \\ Fu-Neng Jiang ${ }^{4}$ \\ Qian-De Liao'
}

'Xiangya Hospital, Central South University, Changsha, Hunan,

${ }^{2}$ Department of Urology, Ningbo No 2 Hospital, Ningbo University School of Medicine, Ningbo, Zhejiang, ${ }^{3}$ Shanghai East Hospital, Tongji University, Shanghai, ${ }^{4}$ Guangdong Key Laboratory of Clinical Molecular Medicine and Diagnostics, Guangzhou First People's Hospital, Guangzhou Medical University, Guangzhou, People's Republic of China
Correspondence: Qian-De Liao Xiangya Hospital, Central South University, Changsha 410008, Hunan, People's Republic of China $\mathrm{Tel}+8617706617315$

Fax +862083373322

Email qiandeliao@I26.com
Objective: Solute carrier family 39, member 14 (SLC39A14), has been identified as a potential biomarker for various cancers. However, its roles in prostate cancer (PCa) are still unclear. The aim of this study was to investigate the clinical significance of SLC39A14 in patients with PCa and its functions in malignant phenotypes of PCa cells.

Patients and methods: Subcellular localization and expression pattern of SLC39A14 protein were examined by immunohistochemistry. Then, the associations of SLC39A14 expression with various clinicopathological features and clinical outcome of patients with PCa were statistically evaluated. Subsequently, the effects of SLC39A14 overexpression and knockdown on PCa cell proliferation and motility were, respectively, examined by Cell Counting Kit-8, transwell, and wound-healing assays.

Results: The immunoreactive scores of SLC39A14 protein in human PCa tissues were significantly lower than those in normal prostate tissues. Based on the Taylor dataset, SLC39A14 downregulation occurred more frequently in patients with $\mathrm{PCa}$ with a higher Gleason score $(P<0.001)$, advanced clinical stage $(P=0.008)$, presence of metastasis $(P=0.009)$, and prostatespecific antigen failure $(P=0.006)$. More interestingly, the survival analysis identified SLC39A14 as an independent factor for predicting the biochemical recurrence-free survival of patients with PCa $(P=0.017)$. Functionally, the enforced expression of SLC39A14 could suppress cell proliferation, invasion, and migration of $\mathrm{PCa}$ cell lines in vitro, which could be reversed by the knockdown of SLC39A14.

Conclusion: Decreased expression of SLC39A14 may lead to malignant phenotypes of PCa cells and aggressive tumor progression in patients with PCa. Importantly, SLC39A14 may function as a tumor suppressor and a biomarker for screening patients with biochemical recurrence following radical prostatectomy.

Keywords: prostate cancer, solute carrier family 39 member 14, biochemical recurrence-free survival, tumor suppressor

\section{Introduction}

As one of the major public health problems, prostate cancer $(\mathrm{PCa})$ represents the most frequent noncutaneous malignancy of the urinary system. ${ }^{1}$ Notably, the incidence and morbidity rate of $\mathrm{PCa}$ have been steadily increasing in recent years. For example, $\sim 241,000$ new cases of PCa were diagnosed in the US in $2011,{ }^{2}$ and the morbidity rate of PCa has increased by 14\% annually since 1990 in the People's Republic of China. ${ }^{3}$ In clinical analysis, the phenotypes of patients with PCa develop from indolent tumors with no or little clinical significance to aggressive metastatic and lethal diseases. ${ }^{4}$ Although many patients with PCa with locally defined disease are often successfully treated with 
surgery and/or radiotherapy and have experienced long-term survival, the biochemical recurrence (BCR), defined as postoperative serum prostate-specific antigen $(P S A) \geq 0.2 \mathrm{ng} / \mathrm{mL}$, which is generally the earliest indicator of recurrent disease, still occurs in an estimated $15 \%-50 \%$ of patients following radical prostatectomy (RP) on long-term follow-up. ${ }^{5}$ Since $\mathrm{PCa}$ is a clinically heterogeneous and multifocal disease, it is a multistep process of influencing progression and prognosis by various molecular mechanisms involving both genetic insults to epithelial cells and changes in epithelial-stromal interactions. ${ }^{6}$ Recent studies have indicated several clinical parameters, such as serum PSA levels, age and underlying health of men, the extent of tumor spread, appearance under the microscope, and the response to initial treatment, to be prognostic markers in the treatment settings. However, there are currently no definitive clinical methods that can reliably predict the responses to clinical therapies for $\mathrm{PCa} .^{7}$ Thus, it is of great clinical significance to identify novel and efficient molecular markers for early risk detection of BCR and for prognosis of patients with localized PCa after surgery.

Solute carrier family 39 (zinc transporter), member 14 (SLC39A14), is a member of the family of zinc transporters that functionally control intracellular zinc influx and efflux from intracellular compartments as well as between the extracellular and intracellular environment. ${ }^{8}$ SLC39A14 encodes Zrt/IRT-like protein 14 (ZIP14), which is a zincand iron-importing protein highly expressed in the liver, pancreas, and heart tissues and also in the adipose tissue. ${ }^{9}$ As a metal transporter, ZIP14 contains multiple transmembrane domains and mediates both nontransferrin- and transferrinbound iron uptake into cells. ${ }^{10}$ Accumulating evidence has recently shown that zinc is essential for cell differentiation and proliferation, and SLC39A14 plays a crucial role in the differentiation of hepatocytes and chondrocytes. ${ }^{11}$ Pathologically, SLC39A14 has been identified as a potential biomarker for lung cancer, gastric cancer, and colorectal cancer. ${ }^{12,13}$ However, its roles in human PCa are still unclear.

To investigate the clinical significance of SLC39A14 in patients with $\mathrm{PCa}$ and its functions in malignant phenotypes of PCa cells, in the current study, we examined the subcellular localization and expression pattern of SLC39A14 protein by immunohistochemistry. Then, the associations of SLC39A14 expression with various clinicopathological features and clinical outcome of patients with PCa were statistically evaluated based on Taylor dataset. In addition, SLC39A14 was upregulated by transient transfection with plasmids and downregulated by transfection with siRNA. The effects of SLC39A14 overexpression and SLC39A14 knockdown on PCa cell proliferation and motility were, respectively, examined by Cell Counting Kit-8 (CCK-8), transwell, and wound-healing assays.

\section{Patients and methods}

\section{Patients and tissue samples}

The study was approved by the Research Ethics Committee of Guangzhou First People's Hospital, People's Republic of China. Informed consent was obtained from all the patients.

All specimens were handled and made anonymous according to the ethical and legal standards.

We used the same patients and tissue samples as in our previous study. ${ }^{14}$ Detailed information of patients and tissue samples is given in the Supplementary materials.

\section{Cell culture and transfection}

Human PCa cell line, LNCaP, was obtained from Guangzhou Land Biosciences Co., Ltd. (Guangzhou, People's Republic of China) and was cultured in RPMI 1640 medium (HyClone Laboratories, Inc., Waltham, MA, USA; Category no SH30809.01B) supplemented with 10\% fetal bovine serum (HyClone Laboratories, Inc.; Category no SH30087.01), $2 \mathrm{mM}$ L-glutamine, and antibiotics. LNCaP cells were maintained at $37^{\circ} \mathrm{C}$ in a humidified chamber supplemented with $5 \% \mathrm{CO}_{2}$. LNCaP cells were transfected with designed siRNA (si-SLC39A14 or si-con) or plasmid (en-SLC39A14 or en-con) with lipofectamine 2000. All siRNAs and plasmids were synthesized by Dharmacon (Lafayette, CO, USA).

\section{Immunohistochemistry}

Expression pattern and subcellular localization of SLC39A14 protein in clinical PCa tissues were detected by immunohistochemistry, and the immunoreactivity scores were calculated as described in our previous study. ${ }^{14}$ Rabbit polyclonal ZIP14 antibody (ProSci incorporated, Poway, CA, USA) was used in this study.

\section{Western blot analysis}

Expression levels of SLC39A14 protein in LNCaP cells were detected by Western blot analysis as described in our previous studies. ${ }^{15,16}$ The antibodies used in this study are listed as follows: rabbit polyclonal ZIP14 antibody (Category no: 6105; ProSci incorporated) and rabbit polyclonal glyceraldehyde 3-phosphate dehydrogenase (GAPDH) antibody (Category no: sc-25778; Santa Cruz Biotechnology Inc., Dallas, TX, USA).

\section{Real-time quantitative reverse transcriptase polymerase chain reaction}

Expression levels of SLC39A14 mRNA in clinical PCa tissues were detected by quantitative reverse transcriptase 
polymerase chain reaction (qRT-PCR) analysis according to the protocol of our previous studies. ${ }^{15,16}$ The sequences of all the primers used in this study are shown as follows: for SLC39A14, forward, 5'-GTC TGG CCT TTG GCA TCC T- $3^{\prime}$, reverse, 5'-AGG GAA CAT ATC AGC CAG AGA AAT-3'; for GAPDH, forward, 5'-ACC CAC TCC TCA CCT TTG A-3', reverse, 5'-CTG TTG CTG TAC CAA ATT CGT-3'.

\section{Cell viability assay}

Cell viabilities were detected by CCK- 8 assay as described in our previous studies. ${ }^{15,16}$

\section{Cell invasion and migration assays}

Cell invasion and migration were, respectively, detected by the transwell and scratch wound-healing motility assays as described in our previous studies. ${ }^{15,16}$

\section{Statistical analysis}

Statistical analyses were performed by SPSS for Windows (Version 11.0; SPSS Inc., Chicago, IL, USA). Continuous variables were expressed as $\bar{X} \pm s$. Difference in immunoreactivity scores of SLC39A14 protein between PCa and adjacent benign tissues was conducted using Wilcoxon signed-rank test. The associations between SLC39A14 protein and mRNA expression and various clinicopathological characteristics were evaluated by Fisher's exact test for any $2 \times 2$ tables and Pearson $\chi^{2}$ test for non- $2 \times 2$ tables. KaplanMeier method was used for the survival analyses, and Cox regression analysis was used for the multivariate analysis. Differences were considered statistically significant when the $P$-value was $<0.05$.

\section{Results}

\section{Decreased expression of SLC39AI4 protein and mRNA in human PCa tissues}

SLC39A14 protein was mainly localized in the membrane and cytoplasm of prostate cells in adjacent noncancerous prostate tissues, but weakly expressed in cancer cells in PCa tissues, as shown in Figure 1A and B. The statistical analysis revealed that SLC39A14 protein expression levels in human PCa tissues were significantly lower than those in adjacent noncancerous prostate tissues $(P<0.01$, Figure 1C). Similarly, SLC39A14 mRNA expression levels in human PCa tissues were also lower than those in adjacent noncancerous prostate tissues based on the Taylor dataset

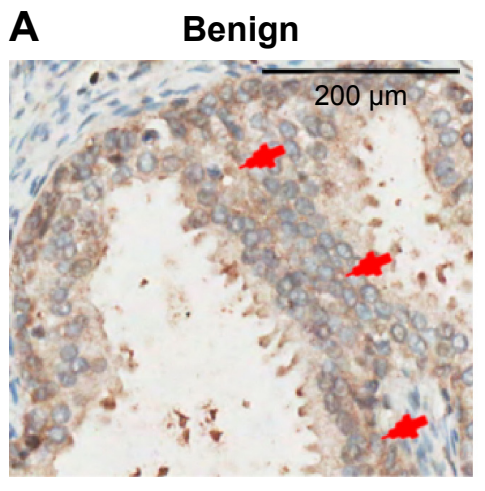

C

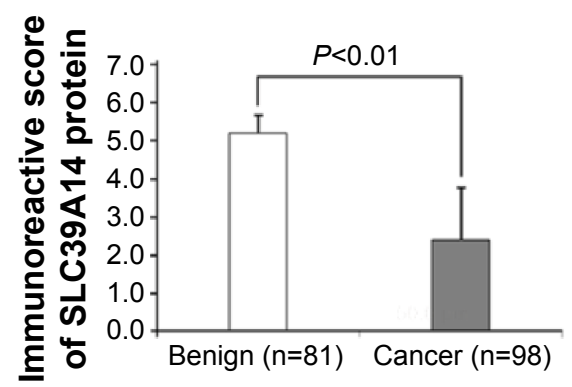

D

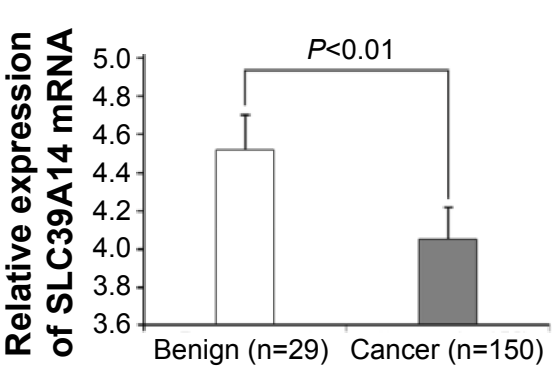

B

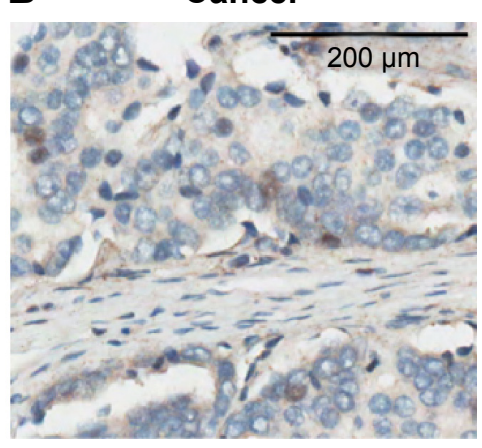

E

Figure I Decreased expression of SLC39A I4 protein and mRNA in human PCa tissues.

Notes: (A) SLC39AI4 protein was mainly localized in the membrane and cytoplasm of prostate cells in adjacent noncancerous prostate tissues. Red arrows show strong positive immunostrainings. Magnification, $\times 400$. (B) SLC39AI4 protein was weakly expressed in cancer cells in PCa tissues. Magnification, $\times 400$. (C) Statistical analysis revealed that SLC39AI4 protein expression levels in human PCa tissues were significantly lower than those in adjacent noncancerous prostate tissues $(P<0.01)$. (D) SLC39AI 4 mRNA expression levels in human PCa tissues were also lower than those in adjacent noncancerous prostate tissues based on the Taylor dataset $(P<0.01)$. (E) SLC39AI4 mRNA expression levels in human PCa tissues were also lower than those in adjacent noncancerous prostate tissues based on our clinical samples $(P<0.01)$. Abbreviations: PCa, prostate cancer; SLC39AI4, solute carrier family 39, member 14. 
$(P<0.01$, Figure 1D) and qRT-PCR analysis based on our clinical PCa samples $(P<0.01$, Figure 1E).

\section{Decreased expression of SLC39AI 4 protein and mRNA associates with the aggressive progression of patients with $\mathrm{PCa}$}

Table 1 summarizes the associations of SLC39A14 protein and mRNA expression with various clinicopathological characteristics based on the results of immunohistochemistry and Taylor dataset. The data revealed that the decreased expression of SLC39A14 protein more frequently occurred in PCa tissues with the higher Gleason score $(P=0.008)$ and the advanced clinical stage $(P=0.012)$. With similar results to the Taylor dataset, SLC39A14 mRNA downregulation occurred more frequently in patients with $\mathrm{PCa}$ with a higher Gleason score $(P<0.001)$, advanced clinical stage $(P=0.008)$, presence of metastasis $(P=0.009)$, and PSA failure $(P=0.006)$.

\section{Decreased expression of SLC39AI 4 mRNA associates with poor prognosis of patients with $\mathrm{PCa}$}

To assess the prognostic value of SLC39A14 mRNA expression in patients with PCa, Kaplan-Meier analysis was performed that showed a significant difference in BCR-free survival $(P=0.017$, Figure $2 \mathrm{~A}$ ), but not in overall survival $(P=0.148$, Figure 2B), between patients with high and low SLC39A14 mRNA expression.

Univariate analysis revealed that SLC39A14 mRNA expression ( $P=0.020$, Table 2$)$, Gleason score $(P<0.001$, Table 2), and pathological tumor stage $(P<0.001$, Table 2$)$ were significant prognostic factors for BCR-free survival in patients with $\mathrm{PCa}$. We then used the Cox proportional hazards multivariate model to examine the association of clinicopathological factors and SLC39A14 mRNA expression with BCR-free survival. The results indicated that SLC39A14 mRNA expression ( $P=0.042$, Table 2$)$, Gleason score $(P<0.001$, Table 2$)$, and pathological tumor stage ( $P=0.012$, Table 2 ) were independent predictors of BCR-free survival in patients with $\mathrm{PCa}$.

\section{Downregulation of SLC39A I 4 promotes proliferation, invasion, and migration of PCa cell lines in vitro}

To detect the cell proliferation, invasion, and migration abilities of LNCaP cells with knockdown of SLC39A14 or reexpression of SLC39A14 (Figure 3A), CCK-8, transwell, and wound-healing assays were performed. CCK-8 assay indicated that the cell viability of LNCaP cells with overexpression of SLC39A14

Table I Association of SLC39A 4 protein and mRNA expression with the clinicopathological characteristics of patients with PCa

\begin{tabular}{|c|c|c|c|c|c|c|}
\hline \multirow[t]{2}{*}{ Clinical features } & \multicolumn{3}{|c|}{ IRS of SLC39A I 4 protein } & \multicolumn{3}{|c|}{ SLC39AI4 mRNA expression } \\
\hline & Number of cases & $\bar{X} \pm s$ & $P$-value & Number of cases & $\bar{X} \pm s$ & $P$-value \\
\hline \multicolumn{7}{|l|}{ Age (years) } \\
\hline$<60$ & 26 & $4.28 \pm I .77$ & 0.426 & 93 & $8.13 \pm 0.44$ & 0.395 \\
\hline$\geq 60$ & 72 & $4.19 \pm 1.26$ & & 57 & $8.07 \pm 0.52$ & \\
\hline \multicolumn{7}{|l|}{ Serum PSA (ng/mL) } \\
\hline$<10$ & - & - & - & 105 & $8.14 \pm 0.49$ & 0.242 \\
\hline$\geq 10$ & - & - & & 42 & $8.04 \pm 0.45$ & \\
\hline \multicolumn{7}{|l|}{ Gleason score } \\
\hline$<8$ & 70 & $4.82 \pm 1.56$ & 0.008 & 22 & $8.19 \pm 0.44$ & $<0.001$ \\
\hline$\geq 8$ & 28 & $3.29 \pm 0.99$ & & 117 & $7.84 \pm 0.45$ & \\
\hline \multicolumn{7}{|l|}{ Clinical stage } \\
\hline$<\mathrm{T} 2 \mathrm{~A}$ & 70 & $4.52 \pm 1.38$ & 0.012 & 80 & $8.24 \pm 0.46$ & 0.008 \\
\hline$\geq \mathrm{T} 2 \mathrm{~A}$ & 28 & $3.65 \pm 0.92$ & & 65 & $8.04 \pm 0.45$ & \\
\hline \multicolumn{7}{|l|}{ Pathological stage } \\
\hline$<\mathrm{T} 3 \mathrm{~A}$ & 70 & $4.28 \pm 1.66$ & 0.206 & 86 & $8.15 \pm 0.42$ & 0.443 \\
\hline$\geq \mathrm{T} 3 \mathrm{~A}$ & 28 & $4.02 \pm 1.18$ & & 55 & $8.09 \pm 0.54$ & \\
\hline \multicolumn{7}{|l|}{ Metastasis } \\
\hline No & 98 & $4.21 \pm 1.68$ & - & 122 & $8.17 \pm 0.44$ & 0.009 \\
\hline Yes & 0 & - & & 28 & $7.86 \pm 0.54$ & \\
\hline \multicolumn{7}{|l|}{ PSA failure } \\
\hline Negative & - & - & - & 104 & $8.19 \pm 0.44$ & 0.006 \\
\hline Positive & - & - & & 36 & $7.94 \pm 0.51$ & \\
\hline
\end{tabular}

Note: "-" indicates there is a lack of relative information of patients in our cohort.

Abbreviations: IRS, immunoreactivity scores; PCa, prostate cancer; PSA, prostate-specific antigen; SLC39AI4, solute carrier family 39 , member 14. 

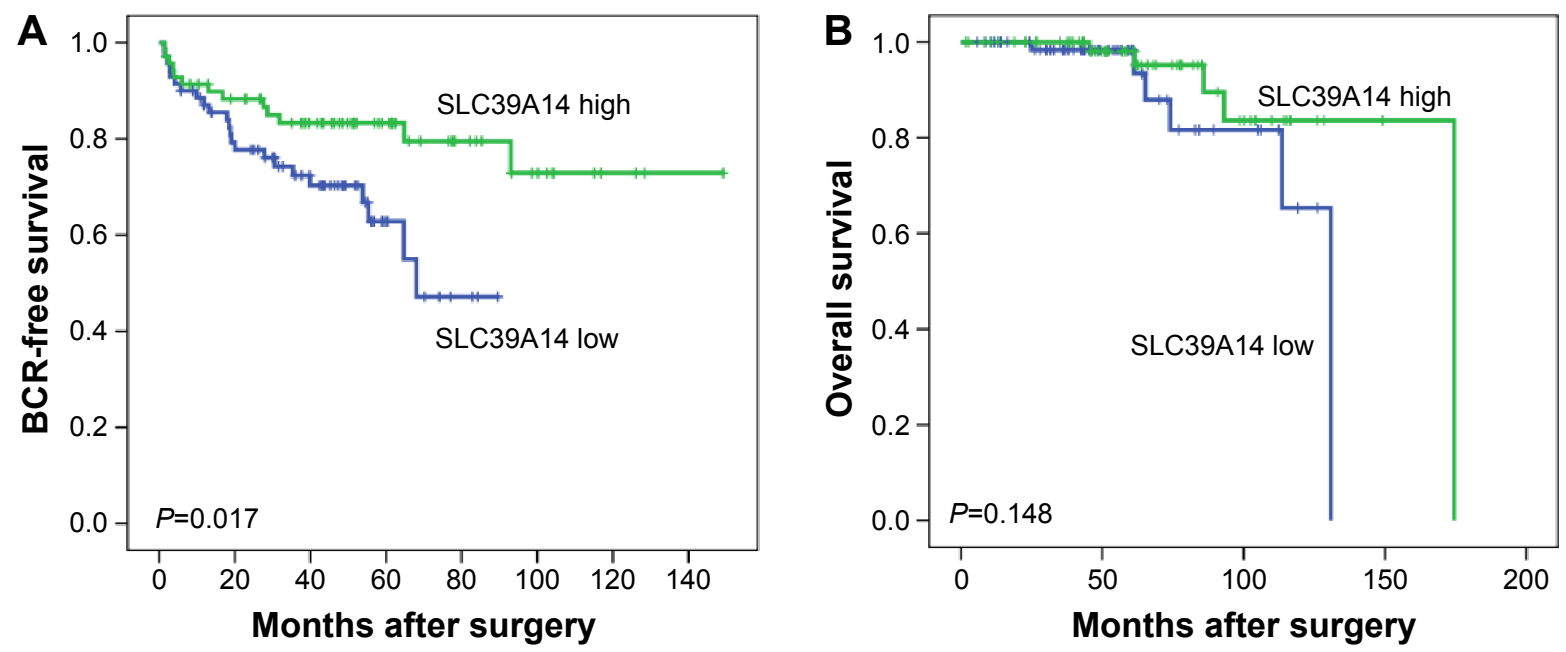

Figure 2 Kaplan-Meier curves of patients with PCa based on SLC39AI4 mRNA expression.

Notes: (A) There was a significant difference in BCR-free survival between patients with high and low SLC39AI4 mRNA expression ( $P=0.017$ ). (B) There was no significant difference in overall survival between patients with high and low SLC39AI4 mRNA expression ( $P=0.148$ ).

Abbreviations: BCR, biochemical recurrence; PCa, prostate cancer; SLC39AI4, solute carrier family 39 , member 14.

was significantly lower than those of control vector-transfected cells (Figure 3B), while knockdown of SLC39A14 dramatically promoted the cell viability (Figure 3C). In addition, transwell assay clearly revealed that the enforced expression of SLC39A14 could effectively inhibit the invasion activity of $\mathrm{LNCaP}$ cells compared to that of control cells at 24 hours after the transfection, while knockdown of SLC39A14 could dramatically enhance the cell invasion (Figure 4). Moreover, wound-healing assay demonstrated that SLC39A14 upregulation could significantly suppress the migration ability of LNCaP cells, while knockdown of SLC39A14 could dramatically enhance the cell migration (Figure 5).

\section{Discussion}

Improved diagnostic and prognostic biomarkers for human $\mathrm{PCa}$ are of great clinical significance to ensure early detection

Table 2 BCR-free survival in univariate and multivariate analysis by Cox regression

\begin{tabular}{lll}
\hline Features & Hazard ratio (95\% CI) & P-value \\
\hline Univariate analysis & & \\
SLC39A I4 mRNA & $2.633(\mathrm{I} .268-6.082)$ & 0.020 \\
Gleason score & $\mathrm{II} .588(5.837-23.008)$ & $<0.00 \mathrm{I}$ \\
Preoperative PSA & $1.312(0.509-3.386)$ & 0.574 \\
Clinical stage group & $1.032(0.530-2.0 \mathrm{II})$ & 0.926 \\
Pathological tumor stage & $5.232(2.564-10.676)$ & $<0.00 \mathrm{I}$ \\
Multivariate analysis & & \\
SLC39A I4 mRNA & $2.182(\mathrm{I} .02 \mathrm{I}-4.38 \mathrm{I})$ & 0.042 \\
Gleason score & $6.94 \mathrm{I}(3.070-15.69 \mathrm{I})$ & $<0.00 \mathrm{I}$ \\
Preoperative PSA & $\mathrm{I} .229(0.457-3.306)$ & 0.683 \\
Clinical stage group & $1.035(0.470-2.283)$ & $0.93 \mathrm{I}$ \\
Pathological tumor stage & $2.888(1.268-6.577)$ & 0.012 \\
\hline
\end{tabular}

Abbreviations: $\mathrm{BCR}$, biochemical recurrence; $\mathrm{Cl}$, confidence interval; PSA, prostate-specific antigen; SLC39A I4, solute carrier family 39, member I4. of aggressive tumors and to initiate appropriate treatment. In the current study, our immunohistochemistry analysis revealed the decreased expression of SLC39A14 protein in human PCa tissues compared to that in normal prostate tissues, which was consistent with the findings of SLC39A14 mRNA based on the Taylor dataset. In addition, SLC39A14 downregulation occurred more frequently in patients with PCa with a higher Gleason score $(P<0.001)$, advanced clinical stage $(P=0.008)$, presence of metastasis $(P=0.009)$, and PSA failure $(P=0.006)$. More interestingly, the survival analysis identified SLC39A14 as an independent factor for predicting the BCR-free survival of patients with $\mathrm{PCa}(P=0.017)$. Functionally, the enforced expression of SLC39A14 could suppress cell proliferation, invasion, and migration of $\mathrm{PCa}$ cell lines in vitro, which could be reversed by the knockdown of SLC39A14. To the best of our knowledge, this is the first study to examine the expression of SLC39A14 in a large cohort of patients with $\mathrm{PCa}$ and to correlate the results with biochemical and pathological values in this malignancy.

As a glycosylated protein located at the plasma membrane and in transferrin-containing endosomal compartments, SLC39A14-encoding protein ZIP14 functions as a metal ion transporter with the capacity to transport divalent cations, such as zinc, iron, and cadmium. ${ }^{17}$ It exists in at least two isoforms that differ from each other in the cation-binding domain. ${ }^{18}$ Growing evidence shows the implications of SLC39A14 in various human malignancies. According to the ONCOMINE database, ${ }^{19}$ SLC39A14 is indicated to be among the top $1 \%$ of genes underexpressed in hepatocellular carcinoma compared to normal liver, which was consistent with the findings of Franklin et al. ${ }^{20}$ The SLC39A14-exon4B 
A

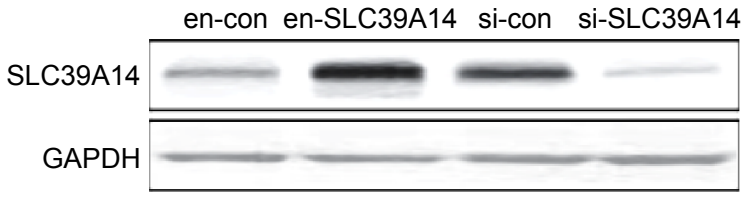

B

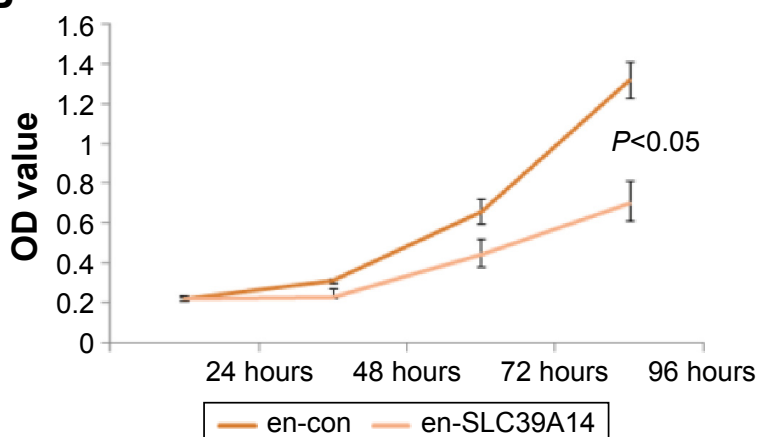

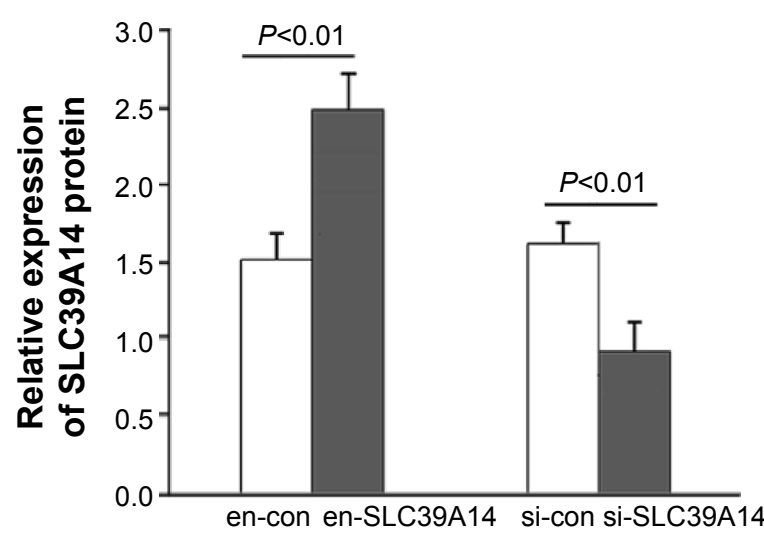

C

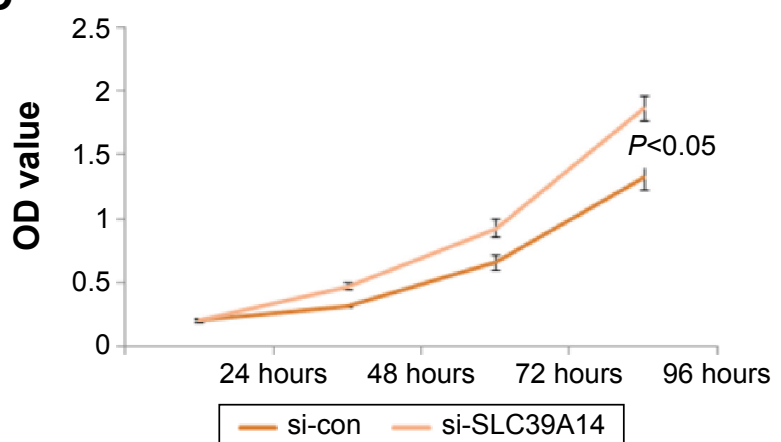

Figure 3 Downregulation of SLC39A I4 promotes cell proliferation of LNCaP cells in vitro.

Notes: (A) Western blot analysis showed that SLC39AI4 protein expression was significantly upregulated by the transfection of SLC39AI4 plasmid (en-SLC39AI4 or encon), but was significantly downregulated by the transfection of SLC39AI4 siRNA (si-SLC39AI4 or si-con). (B) CCK-8 assay indicated that the cell viability of LNCaP cells with overexpression of SLC39AI 4 was significantly lower than those of control vector-transfected cells. (C) CCK-8 assay indicated that the cell viability of LNCaP cells with knockdown of SLC39AI4 dramatically promoted the cell viability.

Abbreviations: CCK-8, Cell Counting Kit-8; GAPDH, glyceraldehyde 3-phosphate dehydrogenase; SLC39AI4, solute carrier family 39, member I4; OD, optical density.
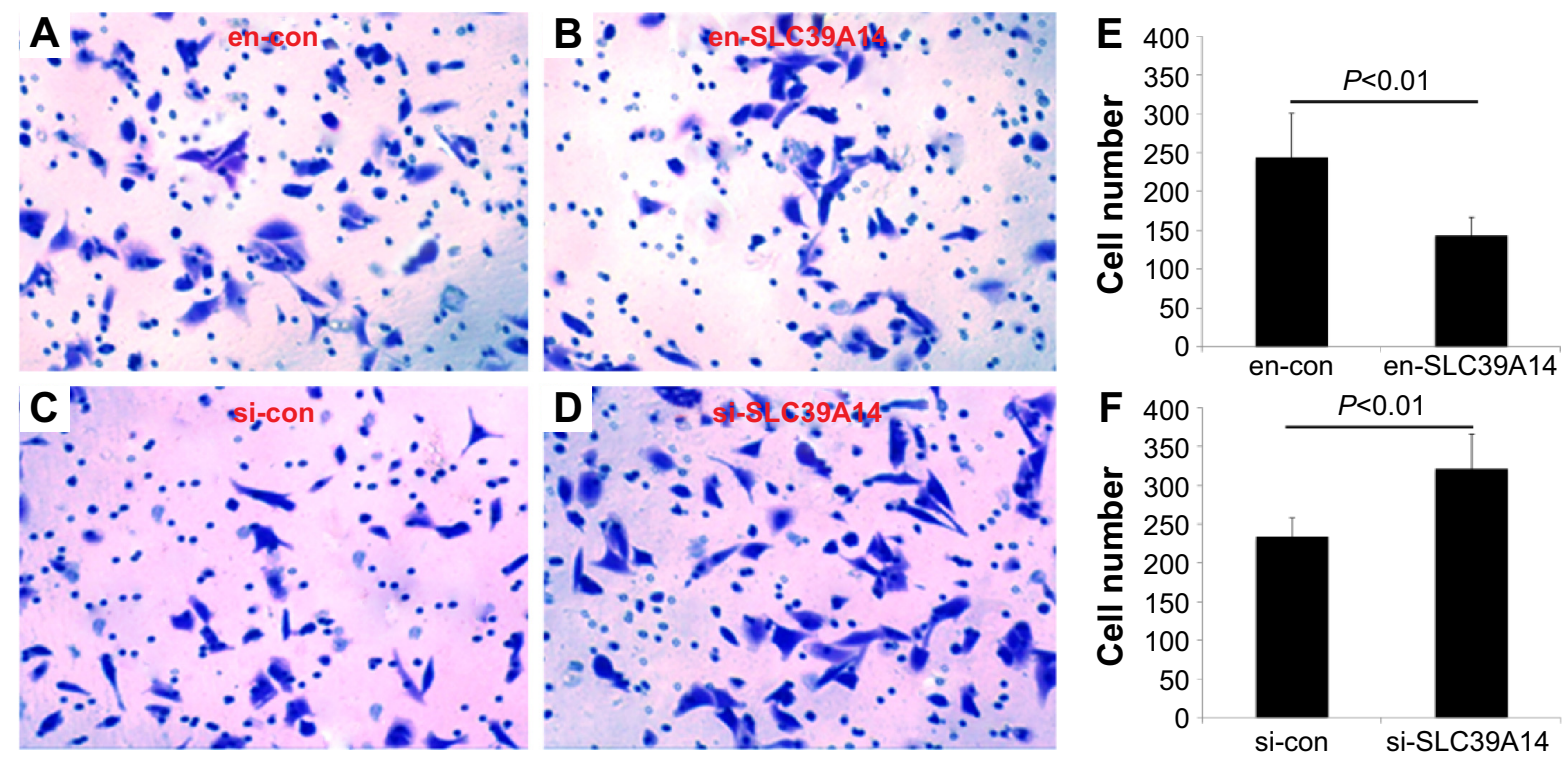

Figure 4 Downregulation of SLC39AI4 promotes invasion of LNCaP cells in vitro.

Notes: Transwell assays clearly revealed that downregulation of SLC39AI4 significantly enhanced the invasion activity of LNCaP cells compared to that of control cells at 24 hours after the transfection, while overexpression of SLC39AI4 dramatically reduced the cell invasion. Cell invasion of LNCaP cells transfected with en-con (A), en-SLC39AI4 (B), si-con (C) and si-SLC39AI4 (D). (E) The number of invasive cells after the transfection of en-con and en-SLC39AI4. (F) The number of invasive cells after the transfection of si-con and si-SLC39AI4.

Abbreviation: SLC39AI4, solute carrier family 39, member 14. 


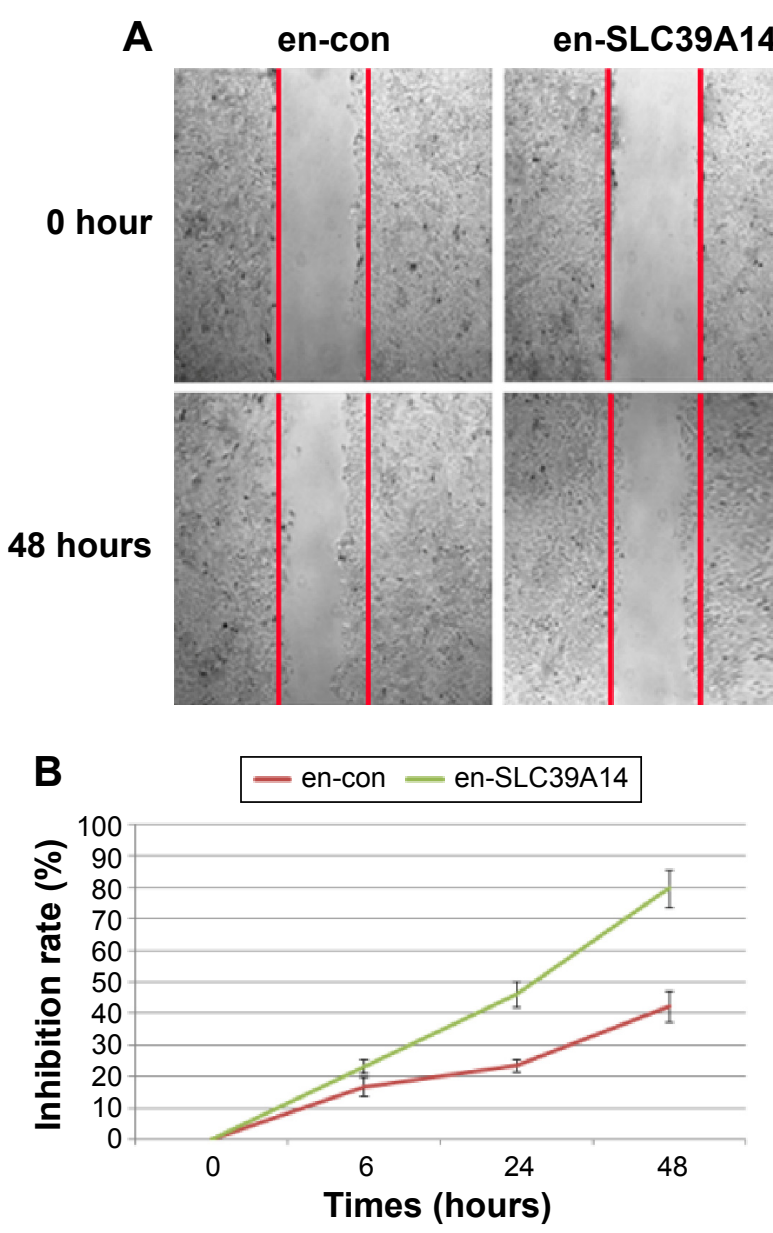

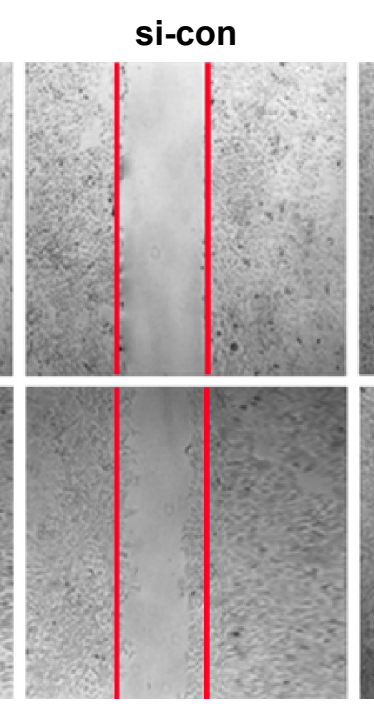
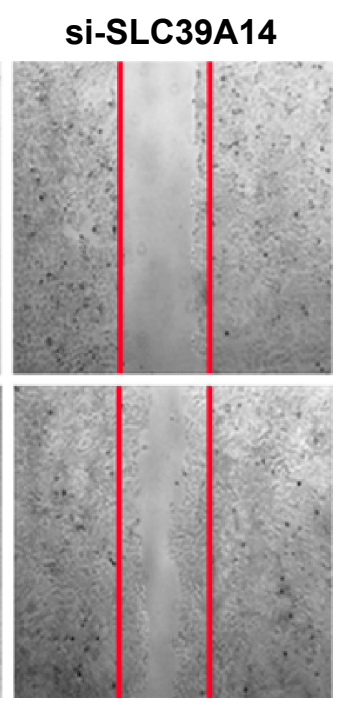

C

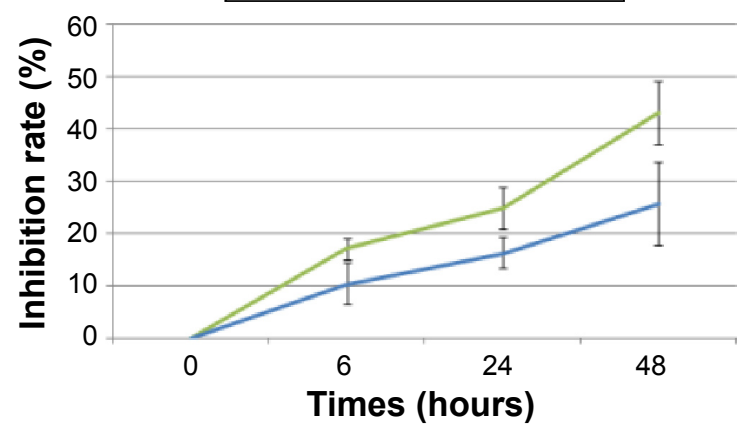

Figure 5 Downregulation of SLC39A I4 promotes migration of LNCaP cells in vitro.

Notes: (A) Cell migration of LNCaP cells transfected with en-con, en-SLC39AI4, si-con and si-SLC39AI4. (B) Inhibition rate of LNCaP cells after the transfection of encon and en-SLC39AI4. (C) Inhibition rate of LNCaP cells after the transfection of si-con and si-SLC39AI4. Wound-healing assays demonstrated that downregulation of SLC39A I 4 significantly enhanced the migration activity of LNCaP cells compared to that of control cells at 24 hours after the transfection, while overexpression of SLC39A I 4 dramatically reduced the cell migration.

Abbreviation: SLC39AI4, solute carrier family 39, member 14.

transcript variants were identified as biomarkers of colorectal, gastric, and lung cancers which are known to have an aberrantly activated WNT-signaling pathway. ${ }^{12,13}$ However, the link between SLC39A14 expression and patients with PCa has not yet been well defined. The current study showed that SLC39A14 mRNA and protein levels, assayed, respectively, by qPCR and immunohistochemistry in prostate tissues, were both lower in PCa tissues than those in adjacent noncancerous prostate tissues. Then, we found that the downregulation of SLC39A14 was significantly correlated with Gleason score, clinical stage, metastasis, and PSA failure, but not with age, serum PSA, and pathological stage. Our further analysis indicated that there was a strong correlation between SLC39A14 expression and patient BCR-free survival. In Kaplan-Meier analysis, we found that patients with PCa with low SLC39A14 expression had a high probability of BCR after RP compared to high SLC39A14 expression groups. Cox regression analysis implied that SLC39A14 was one of the potential independent factors in $\mathrm{PCa}$ after $\mathrm{RP}$, which could act as a prognostic factor for predicting the risk of BCR.

Motility of tumor cells is regarded as a sign of invasiveness and a crucial step in metastasis. ${ }^{21}$ In this study, we investigated the function of SLC39A14 in LNCaP cells using gene overexpression and knockdown techniques. Further experiments presented here showed that the proliferation, migration, and invasion of $\mathrm{PCa}$ cells, following transfection with siRNA-SLC39A14, were obviously promoted compared to the negative control cells, which is contrary to the results following transfection with SLC39A14 plasmid. These results suggest that SLC39A14 may be an important factor for inhibiting cell viability, migratory ability, and invasiveness in $\mathrm{PCa}$ progression, and it may function as a tumor suppressor.

\section{Conclusion}

Our data suggest, for the first time, that the decreased expression of SLC39A14 may lead to malignant phenotypes of PCa 
cells and aggressive tumor progression of patients with $\mathrm{PCa}$. Importantly, SLC39A14 may function as a tumor suppressor and a biomarker for screening patients with BCR following RP. Further studies with a large sample size are needed to confirm these findings.

\section{Acknowledgments}

This study was supported by grants from Natural Science Foundation of Guangdong Province (2014A030310088), Science and Technology Project of Bureau of Health in Guangzhou Municipality (20141A011007), and Projects of Guangdong Key Laboratory of Clinical Molecular Medicine and Diagnostics.

\section{Disclosure}

The authors report no conflicts of interest in this work.

\section{References}

1. Siegel R, Naishadham D, Jemal A. Cancer statistics, 2013. CA Cancer J Clin. 2013;63(1):11-30.

2. Siegel R, Ward E, Brawley O, Jemal A. Cancer statistics, 2011: the impact of eliminating socioeconomic and racial disparities on premature cancer deaths. CA Cancer J Clin. 2011;61(4):212-236.

3. Zhang H, Qi C, Li L, Luo F, Xu Y. Clinical significance of NUCB2 mRNA expression in prostate cancer. J Exp Clin Cancer Res. 2013;32(1):56.

4. Nandana S, Chung LW. Prostate cancer progression and metastasis: potential regulatory pathways for therapeutic targeting. Am J Clin Exp Urol. 2014;2(2):92-101.

5. Molitierno J, Evans A, Mohler JL, Wallen E, Moore D, Pruthi RS. Characterization of biochemical recurrence after radical prostatectomy. Urol Int. 2006;77(2):130-134.

6. Klotz L. Hormone therapy for patients with prostate carcinoma. Cancer. 2000;88(12 suppl):3009-3014.

7. Lapointe J, Li C, Higgins JP, et al. Gene expression profiling identifies clinically relevant subtypes of prostate cancer. Proc Natl Acad Sci USA. 2004;101(3):811-816.
8. Jenkitkasemwong S, Wang CY, Mackenzie B, Knutson MD. Physiologic implications of metal-ion transport by ZIP14 and ZIP8. Biometals. 2012;25(4):643-655.

9. Taylor KM, Morgan HE, Johnson A, Nicholson RI. Structure-function analysis of a novel member of the LIV-1 subfamily of zinc transporters, ZIP14. FEBS Lett. 2005;579(2):427-432.

10. Tominaga K, Kagata T, Johmura Y, Hishida T, Nishizuka M, Imagawa M. SLC39A14, a LZT protein, is induced in adipogenesis and transports zinc. FEBS J. 2005;272(7):1590-1599.

11. Beker Aydemir T, Chang SM, Guthrie GJ, et al. Zinc transporter ZIP14 functions in hepatic zinc, iron and glucose homeostasis during the innate immune response (endotoxemia). PLoS One. 2012;7(10):e48679.

12. Sveen A, Bakken AC, Ågesen TH, et al. The exon-level biomarker SLC39A14 has organ-confined cancer-specificity in colorectal cancer. Int $J$ Cancer. 2012;131(6):1479-1485.

13. Thorsen K, Mansilla F, Schepeler T, et al. Alternative splicing of SLC39A14 in colorectal cancer is regulated by the Wnt pathway. Mol Cell Proteomics. 2011;10(1):M110.002998.

14. Chen JH, Liang YX, He HC, et al. Overexpression of PDZ-binding kinase confers malignant phenotype in prostate cancer via the regulation of E2F1. Int J Biol Macromol. 2015;81:615-623.

15. Chen G, Liang YX, Zhu JG, et al. CC chemokine ligand 18 correlates with malignant progression of prostate cancer. Biomed Res Int. 2014;2014:230183.

16. Lin ZY, Huang YQ, Zhang YQ, et al. MicroRNA-224 inhibits progression of human prostate cancer by downregulating TRIB1. Int J Cancer. 2014;135(3):541-550.

17. Troche C, Aydemir TB, Cousins RJ. Zinc transporter Slc39a14 regulates inflammatory signaling associated with hypertrophic adiposity. Am J Physiol Endocrinol Metab. 2016;310(4):E258-E268.

18. Maxel T, Smidt K, Larsen A, et al. Gene expression of the zinc transporter ZIP14 (SLC39a14) is affected by weight loss and metabolic status and associates with PPAR $\gamma$ in human adipose tissue and 3T3-L1 pre-adipocytes. BMC Obes. 2015;2:46.

19. Rhodes DR, Yu J, Shanker K, et al. ONCOMINE: a cancer microarray database and integrated data-mining platform. Neoplasia. 2004; 6(1):1-6.

20. Franklin RB, Levy BA, Zou J, et al. ZIP14 zinc transporter downregulation and zinc depletion in the development and progression of hepatocellular cancer. J Gastrointest Cancer. 2012;43(2):249-257.

21. Rinker-Schaeffer CW, Chekmareva MA, Mohler JL. The role of motility proteins and metastasis-suppressor genes in prostate cancer progression. Stem Cells. 1996;14(5):508-516. 


\section{Supplementary material \\ Patients and tissue samples}

The study was approved by the Research Ethics Committee of Guangzhou First People's Hospital, People's Republic of China. Informed consent was obtained from all the patients. All specimens were handled and made anonymous according to the ethical and legal standards.

For immunohistochemistry analysis, tissue microarray including 98 primary prostate cancer $(\mathrm{PCa})$ tissues and 81 adjacent noncancerous prostate tissues was obtained from Shanghai Outdo Biotech Co., Ltd. (Shanghai, People's Republic of China; Category no: HPro-Ade180PG-01), along with the detailed clinical information (Table S1). None of the patients recruited in this study had chemotherapy or radiotherapy before the surgery.

For real-time quantitative reverse transcriptase polymerase chain reaction analysis, ten pairs of primary $\mathrm{PCa}$ and self-matched adjacent noncancerous frozen samples were obtained from the tissue bank at Guangzhou First People's Hospital (Table S1). None of the patients recruited in this study received chemotherapy or radiotherapy before the surgery. The pathological diagnosis was performed preoperatively and confirmed postoperatively. All the patients were reviewed, and all the specimens were reexamined in November 2012.

For survival analysis, the results of Taylor dataset, which is a large PCa dataset with microarray expression data for microRNAs and protein-coding genes (mRNAs), and information on the survival of patients including 150 primary PCa tissues and 29 adjacent noncancerous prostate tissues, were also collected. The detailed information on the clinical features of all patients in this study is shown in Table S1.

All 150 patients in the Taylor dataset were given a follow-up exam ranging from 1 month to 175 months (median, 51 months). For the analysis of the survival and follow-up, the date of prostatectomy was used to represent the beginning of the follow-up period. The primary end point of the analysis was biochemical recurrence-free survival. The end point of the other analysis was overall survival. All the patients who died from diseases other than PCa or unexpected events were excluded from the case collection.

Table S I Clinical features of all patients

\begin{tabular}{|c|c|c|c|}
\hline \multirow[t]{2}{*}{ Clinical features } & \multicolumn{3}{|l|}{ Experiment type } \\
\hline & Immunohistochemistry & Taylor dataset & qRT-PCR \\
\hline PCa (number of cases) & 98 & 150 & 10 \\
\hline Mean age (years), mean $\pm S D$ & $70.71 \pm 7.98$ & $58.74 \pm 7.06$ & $68.22 \pm 6.38$ \\
\hline$<60$ & 26 & 93 & 3 \\
\hline$\geq 60$ & 72 & 57 & 7 \\
\hline \multicolumn{4}{|l|}{ Serum PSA levels (ng/mL) } \\
\hline$<10$ & - & 105 & 6 \\
\hline$\geq 10$ & - & 42 & 4 \\
\hline \multicolumn{4}{|l|}{ Gleason score } \\
\hline$\geq 8$ & 70 & 22 & 7 \\
\hline$<8$ & 28 & 117 & 3 \\
\hline Metastasis & 0 & 28 & 0 \\
\hline Adjacent benign tissue (cases) & 81 & 29 & 10 \\
\hline
\end{tabular}

Note: "-" indicates no information was collected.

Abbreviations: qRT-PCR, quantitative reverse transcriptase polymerase chain reaction; PCa, prostate cancer; PSA, prostate-specific antigen.

\section{Publish your work in this journal}

OncoTargets and Therapy is an international, peer-reviewed, open access journal focusing on the pathological basis of all cancers, potential targets for therapy and treatment protocols employed to improve the management of cancer patients. The journal also focuses on the impact of management programs and new therapeutic agents and protocols on patient perspectives such as quality of life, adherence and satisfaction. The manuscript management system is completely online and includes a very quick and fair peer-review system, which is all easy to use. Visit http://www.dovepress.com/testimonials.php to read real quotes from published authors 